\title{
Political Economy Analysis of Intermittent Preventive Treatment of Malaria in Pregnancy Using Sulfadoxine-Pyrimethamine in the Volta Region of Ghana
}

Livingstone Asem ( $\square$ lasem@st.ug.edu.gh )

University of Ghana

Research Article

Keywords:

Posted Date: January 24th, 2022

DOI: https://doi.org/10.21203/rs.3.rs-1254830/v1

License: (c) (i) This work is licensed under a Creative Commons Attribution 4.0 International License. Read Full License 


\section{Abstract \\ Introduction}

Over years, stakeholder control over the implementation process of intermittent preventive treatment of malaria in pregnancy using sulfadoxine-pyrimethamine (IPTp-SP) has been underestimated. As a result, stakeholder influence has not been effectively organized to achieve the global target of $80 \%$ or more for the uptake of 3 plus doses of IPTp-SP. This study aimed to explore the influence of stakeholders on the implementation of IPTp-SP policy.

\section{Methods}

In-depth interviews were conducted with key stakeholders purposively identified in the region. Data was transcribed, coded and themes were developed from the responses using Atlas ti 8.

\section{Results}

The primary actors identified in the implementation of IPTp-SP policy include; interest groups, bureaucracies, financial decision-makers, political leaders, policy beneficiaries, and donors. It was discovered that activities such as the formation of mother support groups help to educate women in their communities to use IPTp-SP policy and soliciting timely feedback from policy recipients positively influences the uptake of the IPTP-SP policy. Whilst stakeholder activities like the failure of the ministry of health to provide practical proof on the possible consequences of IPTp-SP policy, the command-andcontrol tactics of controlling from the national level, the unclear budget delivery, and spending, and uncoordinated donor activities negatively affect the IPTP-SP policy implementation.

\section{Conclusions}

For a successful IPTp-SP policy implementation, implementers must understand and address all rivalry, resistance, and collaboration among the various actors controlling the IPTp-SP policy implementation.

\section{Introduction}

Over the years stakeholder control over the implementation process was undervalued in the IPTp-SP policy implementation and subsequently could not take complete advantage of the positive stakeholder's influence to improve on uptake of IPTp-SP policy. Stakeholder examinations are crucial exercises to guarantee the effective implementation of any health policy. Nevertheless, it was barely done in LMICs including Ghana, thus the need to initiate a process of developing stakeholder examination abilities. As a model process, this research work seeks to respond to the question: What have been the actions and 
inactions of stakeholders that shape the successful implementation of IPTp-SP policy in the Volta Region of Ghana?

Political economy analysis (PEA) was adopted to situate IPTp-SP policy within the understanding of the existing political and economic process in the Volta Region. This framework allows for the examination of the power relationships within a complex network of actors. PEA takes into account limited institutional capacity, risks of elite capture, and the frequent failure of expected collective action (1). Often, what typically prompts to undertake PEA, is that understanding the technical side alone is insufficient to understand the inability to achieve the global target of IPTp-SP policy. Moreover, government counterparts themselves get frustrated by the political economy dynamics such as resistance to reforms from influential stakeholders who stand to lose. They may therefore not appreciate the policy implementers' effort to consider not only the technical side of the solution but also the strategy for making them happen. The challenge that the change teams then face is how to develop the analysis in a way that leads to good quality and operationally useful outputs. This analysis was based on the Campos and Reich (2019) framework which draws on research works from political science and social science whereas being concerned with practicality. The intention is to support individuals assigned with tactical planning for IPTp-SP policy implementation. These individuals comprise government policymakers, top-level implementers, and policy actors outside government. This will also help people responsible for crafting health policy, to predict execution challenges that can be tackled in the design stage before implementation (Campos \& Reich, 2019).

The effective implementation of the IPTp-SP policy is affected by the activities of several individuals as shown in Fig. 1 above. The action and inactions of these individuals may potentially differ from the standard guidelines for implementation thereby compromising the effectiveness of the implementation process. An understanding of the motivations, activities, and power of these actors within the policy environment will provide insight into the nature of the implementation process. Crucially, in most instances, there is a disconnect between policy designers and policy implementers. Often, they are influenced by different perspectives as implementers frequently fail to connect their ideas to the practical exegesis of health care delivery on the ground.

One implementation challenge encountered is that the liability for execution of IPTp-SP policy mostly rests with diverse groups of government officials than the stakeholders who design the health strategy (3). Implementers of these health policy guidelines most times do not fully appreciate the standpoint of the policy designers (3). The IPTp-SP policy process implementation needs work within and across a group of individual stakeholders and stakeholders' organizations to explain strategic purposes of the policy, guarantee resource availability, to fully attain collective ownership of the policy by the policy implementation team, and survive rivalry and continued participation. Thus, for any policy to be well implemented or executed a joint effort and contribution from multiple stakeholders and agencies are required. However, this often ends in long interruptions, negotiation of resources, and misunderstanding between the policy recipients (4). 
To effectively move IPTp-SP policy to full-scale implementation, implementers must genuinely deliberate on the difficulties of executing or implementing the policy in their specific local environment. Health policy implementers are required to know the difficulties and qualities of the organizational context in which the strategies will be implemented. The leaders of IPTp-SP policy implementation need determination, self-control, and diligence to work within a specific environment, and they are required to make robust choices concerning human resources, administrative arrangement, and stakeholder relationships for policy to be well implemented (5).

Health policy implementation often entails an agreement between various individuals, management of divergent views and influence negotiating between various actors situated within the dissimilar setting of the IPTp-SP policy; conglomerates of the financial plan and supervisory groups within government; official and unofficial policy consultants for governmental leaders; the organizations affected and relevant individuals; governmental officials managing the operationalization organizations; administrators with several organizations; and policy recipients including both influential and weak (6). The complex nature of the negotiation procedures required for the full execution of the IPTp-SP policy can affect the adaptation, adjustment, negotiation, substitution or even working against the policy objectives. This led to a literature search on Low-Middle-Income Countries (LMICs) in Google Scholar on the politics of health policy implementation and looking at health policy implementation according to the six tenets of Campos et al. (2019) framework (interest group, bureaucrats, financial decision-makers, political leaders, recipients and external actors) key groups of stakeholders that also share in the IPTP-SP policy implementation. Every group relates to an important category of actors who are concerned with IPTp-SP policy implementation. To explain the different roles of diverse stakeholders' groups, the researcher supported some of the findings with quotes from the key informant interviews on some of the constraints health policy implementers are confronted with and how they can use diverse political tactics to encourage, oppose effective health policy implementation in relation to a particular actor. This research offers a universal appraisal of the political circumstance of effective implementation of IPTP-SP linked to these actors and subsequently deliberated on what way the execution teams can administer these political nuances.

\section{Materials And Methods \\ Study population}

The study population for this qualitative aspect was the interviewing of selected stakeholder group representatives based on their availability and willingness to provide information.

- Regional level: The Regional Director of Health Services, the Regional Public Health Nurse and the Deputy Director of Finance in the Volta Region were included in the interview. All other directors outside the Volta Region were excluded.

- District level: One District Director of Health Services, one coalition of non-governmental organisations in maternal health (AFRINET), one District Manager of the National Health Insurance 
Scheme, 2 providers and 2 service users (clients)

- All other stakeholders not in this position within the region were excluded from the study.

The interview was held from $20^{\text {th }}$ March 2020 to 24 July, 2020.

\section{Selection of study participants}

Purposive sampling was employed in the qualitative component of this study. This was based on a nonprobabilistic procedure where researchers choose the sample based on who they think are appropriate for the study. From this study the position of the stakeholders, their readiness to provide information and their availability at the time of the study was considered for their selection.

\section{Data processing and analysis}

The key informant interviews were audio-recorded using a digital voice recorder and a mobile phone with the permission of the respondent. By audio recording the interviews, the researcher was able to concentrate more fully, listen attentively to what were said. and also observe expressions and other nonverbal cues the interviewees demonstrated during the period of responding. Also, the researcher made brief notes as well in order to be focused $(7,9)$. This allowed questions formulated at the interview to be accurately recorded for use; it also provided accurate and unbiased recording as the interview could be relistened to and allowed direct quotes to be used (9).

After each interview session, the recording was replayed to participants to listen and to make the necessary additions, subtractions and clarifications deemed necessary. Participants listening to tapes after interviews is an important step in qualitative data analysis even though this is often overlooked (10). The taped interviews were transcribed verbatim into Microsoft word.

Analysis of data occurred concurrently with data collection. The process included reading and rereading the interviews and focusing on the identification of themes relating to the explanations and experiences with implementation and uptake of intermittent preventive treatment of malaria in pregnancy using Sulfadoxine-Pyrimethamine.

The research assistants went through the transcript individually multiple times to ensure an improvement in grammar, transcription accuracy and inter-coder reliability. The validated transcripts were further reviewed to enhance understanding of events, categories, and emerging themes. The transcripts were then exported into Atlas ti 8 for data management and analysis. This was followed by the coding process where codes were attached to units of segments of a record. An inductive coding method was used in generating codes and themes. The inductive codes, which included unexpected themes that emerged from the transcripts, were used to generate the various themes. Using the principle of constant comparison, the emerging themes were compared against each other to describe the depth and coverage 
of themes across transcripts. Similar codes were put together and patterns of themes were generated. The assigned codes were organized to generate cross cutting themes from the transcripts (11).

\section{Limitations of the study}

The researcher planned to interview 2 stakeholders from each of the 6 stakeholder groups. Nevertheless, due to unavailability of some of the stakeholders after several follow-ups, only 10 people were reached. However, the researcher interviewed at least one stakeholder from each stakeholder groupings.

\section{Ethical Consideration}

Ethical clearance was obtained from Ghana Health Service's Ethical Review Committee (ERC) with protocol ID No: GHS-ERC 005/12/19 through the University of Ghana, Business School. Approval was given by the Regional Director of Health Service before the study was carried out. The researcher obtained informed consent from each participant before the interview.

All experiments and methods used in the study were performed in accordance with relevant guidelines and regulations. All protocols used in the study were approved by Ghana Health Service Ethical Review Committee. The researcher obtained informed consent from each participant before the interview by completing a consent form.

\section{Results And Discussion}

\section{Interest group politics ("Managing Outside")}

Different interest groups use different strategies to achieve their aim during the implementation of the IPTp-SP policy. In the Volta region, some interest groups frequently attempt to influence the IPTp-SP policy at diverse phases to reduce their costs and expand their benefits from the implementation of the IPTp-SP policy (12). It was found that in the Volta Region, the actors that influence the IPTp-SP policy include health workers, health insurance organizations, health facilities owners, and manufacturing companies of medicines and medical technology.

It is interesting to note that, doctors and midwives are the groups charged with the task of policy implementation, they are usually well structured and more influential than groups service users such as pregnant women who tend to be not well organized and weak. In this study, it was reported that policy recipients or service users are becoming progressively significant with the advancement in various mobile applications (apps) and social media, like the mobile midwife technology implemented by the MOTECH project of Grameen Foundation to provide short messages services (SMS) to the pregnant women about their pregnancy and services expected to be received from the health facilities (13). 
One interesting thing to note is that individuals and their groups use numerous influential tactics to affect health policy implementation. Notably, in the Volta Region, some individuals could apprehend the governing organization liable for the activities of implementing the IPTp-SP policy to gain increased power on how the IPTp-SP policy is executed. This invasion of the state department by interest groups with the attempt to undermine the implementation or structure guidelines to fit their benefit, and also further their personal plans could slow or speed up the implementation of the IPTp-SP policy. Surprisingly, some groups may slow down the implementation of a policy by using their own discretionary powers to exert influence as they engage policy recipients (14).

Now the question is how can an implementation group for IPTp-SP policy resist influences by these parties' concerns? A possible suggestion for this may be that when an influential individual vigorously contests or latently disregards a policy, the implementation group may design a policy to counter the group's influence. It is also possible to initiate activities to organize the interest group to help implement the IPTp-SP policy in the frontline, specifically the district and community level. In some of the instances in the Volta Region when there was a demand on the implementation group to mobilized a fresh organization such as "mother support groups" and "pregnancy school" which has an absolute concern in inspiring execution or organize IPTp-SP service users to performs as an interest group in backing the implementation of IPTp-SP policy. This is supported by the quote below;

"Yes, we do have pregnancy school and mother support groups that help to educate the pregnant women in the communities to take antenatal care seriously" (Health worker 2, 21/05/2020).

Contrary to the expectation, this study did not find any interest group that uses of discretionary influence to resist the implementation of IPTp-SP policy in the Volta region.

\section{Bureaucratic politics ("Managing within and Around")}

Several reports have shown that the main stakeholders tasked with the responsibility for the implementation of IPTp-SP policy are the bureaucrats. Bureaucrats i.e., frontline health workers or streetlevel bureaucrats, are sometimes not involved in the policy development process but however given the responsibility to execute the policy (2). This study found that at the national level there are many state departments with the responsibilities of implementing IPTp-SP policy, creating the problem of interdepartmental teamwork. Rivalry and struggle arise among similar departments to control policies and their implementation, as different departments try to increase their individual power, budget control, human resource, and overall influence on the IPTp-SP policy. This discrepancy in the level of influence, across different state agencies, can interrupt the policy execution, which can be aggravated by budget control politics (15). From our study, rivalry can also happen inside a single agency among divisions and units such as the Nation Malaria Control Program (NMCP) and Family Health Division (FHD) of the Ghana Health Service (2) which negatively affect the implementation of the IPTp-SP policy.

One of the issues that emerged from the findings is that effective Implementation of IPTp-SP policy also depends on the activities of frontline health workers. These frontline health workers become de facto 
frontline policymakers because of the utilization of unrestricted power for alteration, prevention, or endorsement of the supply of IPTp-SP policy benefits to policy recipients. Hence, this explains how in practice the IPTp-SP policy is delivered to pregnant women. This is corroborated by earlier findings from the work done by Lipshy that reported that frontline health service providers actually convert policy aims into action, persuading the lived experience of pregnant women (16). This is supported by the quote below;

"the midwives are the frontline health providers trained to provide the IPTp-SP services to the pregnant women and they follow them up until they deliver" (Regional Director 22/05/2020).

Also, the command-and-control tactics of controlling from the center present a challenge because the health system cannot be directed from the center. This study supported evidence from previous observations by Gilson, that recommends the distribution of leadership at every level of the health system with the objective of using the flow of energy and power to gather the wide array of actors at a different level of the health system to achieve full implementation of IPTp-SP policy (17).

However, it also discovered that to successfully implement IPTp-SP policy, further research should be undertaken by implementation teams to discover common grounds that transverse various actors so they can build trust and credibility (2).

\section{Budget politics ("Managing Money")}

The current study found that the key bottlenecks in IPTp-SP policy execution are the inadequate budgetary allocation of resources for the activities necessary to effectively execute the IPTp-SP policy. This corroborates with earlier works done by Gomez-Dantes and colleagues, who reported that disbursement of resources and its fairness is one of the recurrent concerns which make the budgeting activity complex (18). The allocation of resources by the Ministry of Finance $(\mathrm{MOH})$ to various departments with varied needs also presents a challenge. These financial resources are disbursed from the national level through regional and districts to the various health facilities as shown in the quote below:

"Usually, donor partners put their resources together and pass it through the Ministry of Health, who also allocates it to the Ghana Health Service (GHS). The GHS also have their way of allocating it to regions and districts, these decisions are being made at the national level. The region then will prepare a budget and disburse the funds to the districts according to the national guidelines." (Regional Accountant, 22/05/2020).

The field interviews also point out that the Ministry of Health $(\mathrm{MOH})$ repeatedly failed in providing convincing practical proof on the possible economic consequences of IPTp-SP policy. Additionally, in some of the cases voting of political leaders drives them to promise over ambitious health plans that may not have adequate funding means to see to their execution. A typical example is an announcement by the late President, Prof. JEA Mills on a one-time premium for national health insurance during the 2008 election which was never implemented due to the nonavailability of funds to support the policy. 
Furthermore, budget delivery and spending are mostly not clear or satisfactorily comprehended. For the implementation of the IPTp-SP policy to yield the needed high impacts, the principles of determining the budgets and their disbursement must be transparent and well explained to all the stakeholders (18).

This finding has important implications for the effective implementation of IPTp-SP policy. Thus, effective implementation cannot be achieved without teaming up with the finance ministry and the board that supervises budget preparation and endorsement.

\section{Leadership politics ("Managing Up”)}

A strong relationship between leadership politics and the effective implementation of health policy has been reported in the literature. One interesting finding from the field interviews was that the obligation and capability of leadership to the implementation of IPTP-SP policy greatly influenced its acceptance and full-scale implementation. These findings is consistent with that of Frenk who reported that the health systems want leaders with tactical foresight, practical understanding, influential abilities, and moral direction to move the process of policy implementation into action (19). To overpower the many countless sources of resistance to change involves dynamic leadership with strong organizational abilities. The results further discovered that only a few managers have the know-how as the leaders of a big organization and sometimes they rarely understand the significance of organization abilities (20). The quotes below show some of the management functions;

"For monitoring and supervision, the national does the supervision to the various districts to see what is going on. Aside from regional also does periodic supervision and districts also follow up to the CHPS zones, and at the facility level, we have midwives and CHN who are in-charges that see to these things" (Regional Director).

According to Gilson, for effective IPTp-SP policy implementation, managers must encourage, facilitate, and help in making decisions at each point of the health structure. In accordance with the present results, previous studies have also demonstrated that IPTp-SP policy is obstructed by the frontiers of service provision and during community mobilization (17). In a decentralized political system like Ghana, the center has restricted power to encourage valuable accomplishment at front-line stages. It is possible, therefore, that implementation teams need to engage and marshal senior, and local political actors in order to guarantee a full-scale implementation of the IPTp-SP policy.

\section{Recipients' politics ("Managing Down")}

Several reports have shown that in order to effectively implement the IPTp-SP policy, implementation teams need to discuss how the policy will improve on the current benefits (21). Another important finding from this study was that some policy recipients might perceive that their benefit could be restricted; others might perceive that their profits could increase. To fully operationalize IPTp-SP intervention, it is important to foster confidence with new service users, get their views and occasionally marshal them into the accomplishment of the policy. Policy recipient's opinions concerning IPTp-SP policy could be 
manipulated through contending notions of other groups, which can be a challenge. This can be curtailed by timely and consistent communication with policy recipients (2).

Another most interesting results of this study according to Roberts et al. (2006) was that the health organization has an opportunity to appeal to policy recipient groups to increase the provision of IPTp-SP policy and achieve improved health outcomes (20). The beneficiaries may also be encouraged to participate in planning meetings, to help promote their needs and interests. They also provide feedback on the implementation to the service providers which help in making the necessary adjustment for improvement. The quote is provided below;

"Most of the health facilities have a suggestion box for soliciting feedback from the service users and also do routine client satisfaction surveys to get their views for improvement" (Regional Director).

It was also reported that to improve health outcomes as well as uptake of IPTp-SP policy, you will need a strong institutional motivation, communal engagement to support direct answerability interactions between the IPTp-SP service user, the health workers, and the state (22). In the Volta Region, new opportunities exist with the advent of digital technologies for beneficiary engagement such as using mobile applications (apps) like the MOTECH (Mobile Midwife) implemented in North Tongu and South Dayi of the Volta Region can serve as a strong platform for updating policy recipient on their expectations and benefits, accessing basic information on the policy; offering opportunity to hold health providers liable; educating policy constituents; or developing a system of easily accessible compliant and feedback.

\section{Donor politics ("Managing Externally")}

A strong relationship between donor politics and the effective implementation of IPTp-SP policy was reported in the literature over the years. It was found that actualizing the implementation of IPTp-SP policy includes handling numerous external stakeholders, comprising bilateral aid institutions, multilateral organizations, and international financial organizations, in addition to outside non-country stakeholders like non-state organizations and private for-benefit organizations. The results of the study indicate that the power that donors exert on IPTp-SP policy activities owing to their perceived stronger technical capability and power over funding sources creates numerous challenges, but can also bring good expectations (23). A few of these problems consist of dominating countries' programs, neglecting the capabilities of the country's health care systems, providing terrible direction centered on philosophy or terrible encounters in other nations, and disrupting the country's plan (23).

"Most of the donors come with their own activities and budget and sometimes their own human resource to train the providers" (Regional Director).

This feedback from the director corroborates earlier findings reported by Chan et al (2011) (23). The politics of donors have also been identified to influence the implementation of the IPTp-SP policy process positively. Lately, there has been a drift concerning condensed conditionality on financing and development in straight budgetary support, to guarantee that donor programs augment positively the 
nationwide agenda (24). This is believed to intensify health sector synchronization, develop country ownership and country-wide control. To drive full benefits from donors, policymakers must coordinate the activities of donors to harness their full contribution to the execution of IPTp-SP policy in such a way that it will be coherent with state priorities and subsequently will last after donor resources cease.

\section{Conclusions}

This research set out to determine the influence of stakeholders on the implementation of IPTp-SP policy in the Volta Region of Ghana to direct policy into practical action more successfully. Ghana after the WHO 2012 IPTp-SP policy upgrade, uptake of the policy is still far below the global target of $80 \%$, thus a political examination of the positions, control, and significance of the actors concerned about IPTp-SP policy execution will contribute to explain their roles in encouraging or opposing the execution of IPTp-SP policy in Volta Region of Ghana.

It was discovered that for successful IPTp-SP policy implementation, implementers must understand and address all rivalry, resistance, and collaboration among stakeholders controlling the execution activities. It was also reported that orderly and constant political examination of stakeholders involves in the IPTp-SP policy space can help decision-makers and influential implementers to advance the possibilities for effective policy planning and execution.

Furthermore, it is significant to acknowledge that some encounters in the IPTp-SP policy implementation may be as a result of poor design of the IPTp-SP policy, purposely or accidentally. For example, during antenatal service, all pregnant women are supposed to have an active health insurance card to access the policy for free. This can delay some of them from coming to the antenatal clinic on time to access the service because they do not have active health insurance cards.

Finally, this research recognizes the diverse actors, problems, and outlines examples of practical procedures to achieve a successful IPTp-SP policy implementation that will eventually increase the uptake. There is also a need to widen on the approaches existing to policy implementers to manage actors who could oppose or counter IPTp-SP policy execution and enhance plans for controlling those who help or endorse the execution.

\section{Declarations}

\section{Ethics approval and consent to participate}

Ethical clearance was obtained from Ghana Health Service's Ethical Review Committee (ERC) with protocol ID No: GHS-ERC 005/12/19 through the University of Ghana, Business School. Approval was given by the Regional Director of Health Service before the study was carried out.

\section{Guideline's statement}


All experiments and methods used in the study were performed in accordance with relevant guidelines and regulations. All protocols used in the study were approved by Ghana Health Service Ethical Review Committee. The researcher obtained informed consent from each participant before the interview by completing a consent form.

\section{Consent for publication}

Not Applicable

\section{Availability of data and materials}

All the data material is available in the report

\section{Competing interests}

The authors declare that they have no competing interest

\section{Funding}

This research is self-funded project

\section{Authors' contributions}

LA developed and implement the study, LA conducted the interviews and analyzed the data, and LA draft the manuscripts

\section{Acknowledgments}

I will like to acknowledge Prof. Abdul-Gafaru Abdulai and Dr. Gordon Abeka-Nkrumah who helped in reviewing the content of this paper.

\section{Data availability and materials}

All data generated or analysed during this study are included in this published article

\section{References}

1. Buur L, Baloi O, Mondlane Tembe $C$. Mozambique synthesis analysis: between pockets of efficiency and elite capture. JSTOR; 2012.

2. Campos PA, Reich MR. Political analysis for health policy implementation. Heal Syst Reform. 2019;5(3):224-35.

3. Oliver TR. The politics of public health policy. Annu Rev Public Heal. 2006;27:195-233.

4. Byers $\mathrm{V}$. The challenges of leading change in health-care delivery from the front-line. J Nurs Manag. 2015;1-8. 
5. Pülzl H, Treib O. Implementing public policy. In handbook of public policy analysis. Routledge; 2017. p. 115-34.

6. Mthethwa RM. Critical dimensions for policy implementation. 2012;

7. Batra R, Menz S. Research Methodology: Assessing the potential in adopting a PPP approach to analyze Economic Performance of projects. ARCOM Dr Work Res Methodol. 2015;(April 2015):1-84.

8. Saunders M, Lewis ATP. Research Methods for Business Students Fifth Editon. Pearson, F. 2009. 649 p.

9. Oliveira MA, João J, Ferreira P. Facilitating qualitative research in business studies: Using the business narrative to model value creation. African J Bus Manag. 2011;5(1):68-75.

10. Hardy M, Bryman A. Handbook of data analysis. 2004.

11. Attride-Stirling J. Thematic networks: An analytic tool for qualitative research. Qual Res. 2001;1(3):385-405.

12. Sheikh K, Porter JDH. Disempowered doctors? A relational view of public health policy implementation in urban India. Health Policy Plan. 2011;26(1):83-92.

13. Altman D. Power \& community. Routledge; 2013.

14. Stigler JG. The theory of economic regulation. Routledge; 2021. p. 67-81.

15. World Health Organization. Updated WHO Policy Recommendation. Intermittent preventive treatment of malaria in pregnancy using sulfadoxine-pyrimethamine (PTp-SP). Geneva; 2012.

16. Lipsky M. Street-level bureaucracy: Dilemmas of the individual in public service. Russell Sage Foundation; 2010.

17. Gilson L. Everyday politics and the leadership of health policy implementation. Heal Syst Reform. 2016;2(3):187-93.

18. Gómez-Dantés O, Reich MR, Garrido-Latorre F. Political economy of pursuing the expansion of social protection in health in Mexico. Heal Syst Reform. 2015;1(3):207-16.

19. Frenk J. The global health system: Strengthing Nationa Health System as the next step for global progree. PLoS Med. 2010;7(1):e1000089.

20. Roberts MJ, Hsiao W, Berman P, Reich MR. Getting health reform right: A guide to improving performance and equity. Bull World Health Organ. 2006;84(2):332.

21. Harvey G, Kitson A. PARIHS revisited: from heuristic to integrated framework for the successful implementation of knowledge into practice. Implement Sci. 2015;11(1):1-13.

22. WHO Global Malaria Programme. Intermittent Preventive Treatment of malaria in pregnancy using Sulfadoxine-Pyrimethamine (IPTp-SP). Updated WHO Policy Recommendation. 2012; (October2012):1-2.

23. Chen L, Haan A De, Zhang X, Warmerdam W. Addressing vulnerability in an emerging economy: China 's New Cooperative Medical Scheme (NCMS). Routledge. 2011;(November 2014):37-41.

24. Niyonkuru F. Failure of foreign aid in developing countries: A quest for alternatives. Bus Econ J. 2016;7(3):1-9. 
Figures

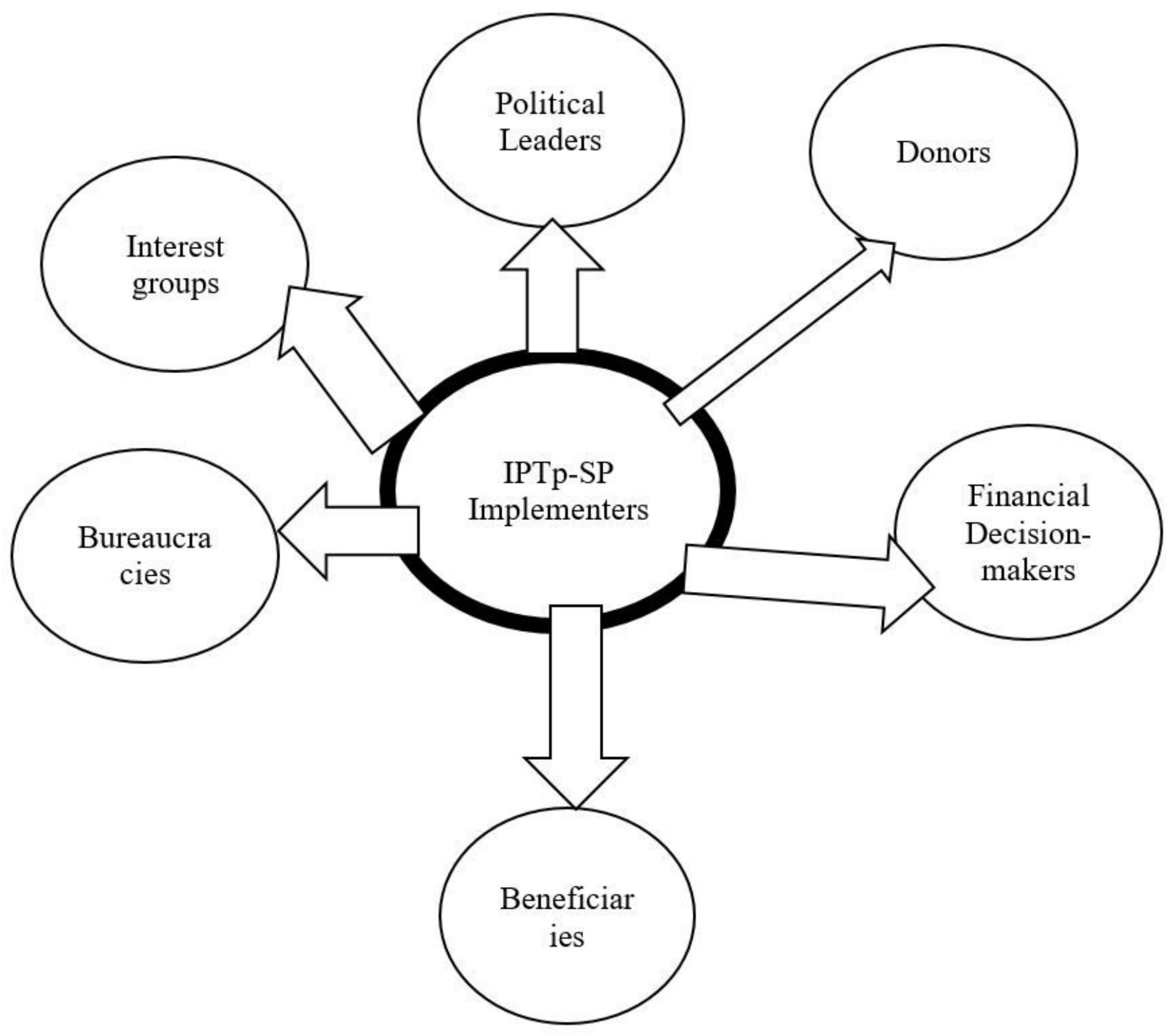

Figure 1

IPTp-SP Policy Implementation Stakeholder, adopted from Campos and Reich 2019 